\title{
Influência da incorporação de polidextrose como substituto de gordura na qualidade de pão de queijo
}

\author{
Effect of replacing fat with polydextrose on quality of cheese \\ bread
}

\author{
Ana Claudia Nunes Palmeira Alexandre ${ }^{1 *}$ (1), Carolina de Souza Salgado1, \\ Willian Rafael Gonçalves Soares ${ }^{1}$, Lúcia Kazue Nakahata Medrado', \\ Elizabeth Harumi Nabeshima² ${ }^{\mathbb{D}}$, Luciana Miyagusku¹, Elisvânia Freitas dos Santos ${ }^{1}$ \\ ${ }^{1}$ Universidade Federal de Mato Grosso do Sul (UFMS), Faculdade de Ciências Farmacêuticas, Alimentos e \\ Nutrição, Campo Grande/MS - Brasil \\ ${ }^{2}$ Instituto de Tecnologia de Alimentos, Cereal Chocotec, Campinas/SP - Brasil
}

*Corresponding Author: Ana Claudia Nunes Palmeira Alexandre, Universidade Federal de Mato Grosso do Sul (UFMS), Faculdade de Ciências Farmacêuticas, Alimentos e Nutrição, Av. Costa e Silva, s/n, CEP: 79070-900,

Campo Grande/MS - Brasil, e-mail: anaclaudia.nunes.alexandre@gmail.com

Cite as: Alexandre, A. C. N. P., Salgado, C. S., Soares, W. R. G., Medrado, L. K. N., Nabeshima, E. H., Miyagusku, L., \& Santos, E. F. (2020). Effect of replacing fat with polydextrose on quality of cheese bread. Brazilian Journal of Food Technology, 23, e2019107. https://doi.org/10.1590/1981-6723.10719

\begin{abstract}
Resumo
Um dos grandes desafios do desenvolvimento de produtos com baixos teores de gordura é manter suas características sensoriais. A polidextrose pode ser utilizada como um substituto de gordura, sem causar grandes alterações no produto final. O objetivo deste estudo foi avaliar as características físico-químicas, propriedades nutricionais e investigar o potencial de aceitação de pães de queijo formulados com polidextrose. Foram elaboradas 4 formulações: formulação 1 (sem substituição de óleo por polidextrose); formulações 2, 3 e 4 (contendo diferentes concentrações de substituição de óleo e polidextrose). Participaram da avaliação sensorial 100 provadores não treinados, de 18 a 62 anos. Nas análises físico-químicas foram determinados os teores de água, cinzas, proteínas, lipídeos, textura, volume específico e cor, sendo realizado cálculo teórico para quantificação de carboidratos (por diferença) e fibras. Os resultados demonstraram que nos atributos avaliados as formulações obtiveram aceitabilidade superior a 70\%, exceto o atributo intenção de compra da formulação padrão. Foi observado que o desenvolvimento de formulações contendo $4 \%$ de polidextrose apresentaram textura semelhante à formulação padrão. Assim, a adição de polidextrose em pães de queijo permitiu a obtenção de produtos com menores teores de lipídeos, maior teor de fibras, boa aceitação sensorial e características tecnológicas semelhantes ao padrão, indicando seu potencial de comercialização.
\end{abstract}

Palavras-chave: Prebiótico; Alimento funcional; Fibra alimentar; Análise sensorial; Nutrição; Lipídeos.

\section{Abstract}

One of the great challenges of developing low-fat products is to maintain their sensory characteristics. Polydextrose can be used as a fat substitute without causing major changes in the final product. The objective of this study was 
to evaluate the physicochemical characteristics, nutritional properties and acceptance of cheese breads made with polydextrose. Four formulations were elaborated: formulation 1 (without substitution of oil for polydextrose); formulations 2, 3 and 4 (containing different concentrations of oil substitution and polydextrose). A total of 100 untrained testers aged 18-62 participated in the sensory evaluation. In the physicochemical analyzes the water moisture, ash, protein, fat, texture, specific volume and color contents were determined. Theoretical calculations were performed for the quantification of carbohydrates (by difference) and fibers. The results showed that for the attributes evaluated, acceptance of the formulations was higher than $70 \%$, except for intention to purchase of the standard formulation. It was observed that the development of formulations included $4 \%$ polydextrose, with texture similar to the standard pattern. Thus, an addition of polydextrose in cheese breads, allows the prodution of products with lower lipid content, higher fiber content, good sensory acceptance and specific technological characteristics for the standard, which shows its commercialization potential.

Keywords: Prebiotic; Functional food; Dietary fiber; Sensory analysis; Nutrition; Fats.

\section{Introdução}

Os consumidores têm buscado alimentos que, além de nutritivos, ofereçam benefícios à saúde e melhora na sua qualidade de vida. Para satisfazer esse novo perfil alimentar, a indústria de alimentos tem realizado grandes avanços com a utilização de ingredientes funcionais (Pinto \& Paiva, 2010). A polidextrose é um ingrediente funcional, sendo classificada como fibra dietética solúvel em água, não hidrolisada pelas enzimas digestivas de humanos, porém é fermentada no cólon (Achour et al., 1994).

Seu efeito prebiótico estimula o crescimento das bactérias benéficas ao trato gastrointestinal e ajuda na redução dos níveis de colesterol e glicose no sangue. Possui baixo valor calórico (1 Kcal/g), potencial para a redução da constipação e aumento da absorção de minerais, e por isso torna-se um ingrediente em destaque para adição em novos produtos, dentre eles pão de queijo (Santos et al., 2010; Fernandes et al., 2015; Ibarra et al., 2015; Carmo et al., 2016).

O pão de queijo é um alimento de ampla aceitação pela população brasileira, além de ser um alimento alternativo para os celíacos, pacientes alérgicos às proteínas do trigo, por ser isento de glúten (Machado \& Pereira, 2010). Atualmente é possível o desenvolvimento de formulações mais saudáveis mantendo características sensoriais esperadas pelo consumidor, como a crocância da casca, a maciez no seu interior, a cor amarelada, além do odor e aroma provenientes do queijo (Diório et al., 2018).

O objetivo deste trabalho foi elaborar e avaliar as características físico-químicas de pães de queijo formulados com adição de polidextrose, com menor teor de gordura em relação ao convencional, bem como investigar seu potencial de aceitação e propriedades nutricionais.

\section{Material e métodos}

O preparo das formulações, as análises microbiológicas, sensorial, físico-químicas, volume específico e estatística foram realizadas na Unidade de Tecnologia de Alimentos e Saúde Pública (UTASP), da Universidade Federal de Mato Grosso do Sul (UFMS), em Campo Grande (MS), e as análises de textura e cor foram realizadas no Laboratório do Cereal Chocotec do Instituto de Tecnologia de Alimentos (ITAL), da Secretaria de Agricultura e Abastecimento do Estado de São Paulo, em Campinas (SP). A análise sensorial foi realizada conforme o parecer do Comitê de Ética em Pesquisa da UFMS de número 1.614.079.

\subsection{Preparo das formulações}

Os ingredientes básicos para as formulações foram adquiridos em supermercados do município de Campo Grande (MS), enquanto a polidextrose foi adquirida na empresa Empreendimento Comercial e Industrial Ecil 
Ltda., São Paulo (SP). Os ingredientes foram pesados em balança digital (Filizola, Brasil) com precisão de $0,1 \mathrm{~g}$ e capacidade máxima de $15 \mathrm{~kg}$.

Foram elaboradas 4 formulações distintas de pão de queijo: F1 = Formulação padrão $(0 \%)-$ sem substituição por polidextrose; F2, F3 e F4 contendo diferentes concentrações de polidextrose, adicionadas em substituição ao óleo de soja como demonstrado na Tabela 1.

Tabela 1. Formulação dos pães de queijo.

\begin{tabular}{ccccc}
\hline \multirow{2}{*}{ Ingredientes } & $\mathbf{F 1}$ & $\mathbf{F 2}$ & $\mathbf{F 3}$ & $\mathbf{F 4}$ \\
\cline { 2 - 5 } & $\mathbf{g ~ ( \% )}$ & $\mathbf{g ~ ( \% )}$ & $\mathbf{g ~ ( \% )}$ & $\mathbf{g}(\mathbf{\%})$ \\
\hline Polvilho doce & $492,0(40,2)$ & $492,0(40,2)$ & $492,0(40,2)$ & $492,0(40,2)$ \\
\hline Polidextrose & - & $24,5(2,0)$ & $49,0(4,0)$ & $73,5(6,0)$ \\
\hline Queijo parmesão & $250,0(20,4)$ & $250,0(20,4)$ & $250,0(20,4)$ & $250,0(20,4)$ \\
\hline Leite integral & $250,0(20,4)$ & $250,0(20,4)$ & $250,0(20,4)$ & $250,0(20,4)$ \\
\hline Óleo de soja & $110,2(9,0)$ & $85,7(7,0)$ & $61,2(5,0)$ & $36,7(3,0)$ \\
\hline Ovos & $110,2(9,0)$ & $110,2(9,0)$ & $110,2(9,0)$ & $110,2(9,0)$ \\
\hline Sal & $12,5(1,0)$ & $12,5(1,0)$ & $12,5(1,0)$ & $12,5(1,0)$ \\
\hline Total (g/\%) & $1.225(100)$ & $1.225(100)$ & $1.225(100)$ & $1.225(100)$ \\
\hline
\end{tabular}

F1 (Padrão): $0 \%$ de polidextrose. F2: $2 \%$ de polidextrose. F3: $4 \%$ de polidextrose. F4: $6 \%$ de polidextrose.

\subsection{Processo de fabricação dos pães de queijo}

As amostras foram preparadas pelo método de escaldamento, segundo Pereira et al. (2004), utilizando na formulação padrão polvilho doce (Donana ${ }^{\circledR}$ ) e o polvilho doce com polidextrose (formulações 2,3 e 4). Inicialmente foi realizado o preparo da solução de escaldamento composta de leite integral (Piracanjuba ${ }^{\circledR}$ ), óleo de soja (Liza $\left.{ }^{\circledR}\right)$ e sal (Cisne $\left.{ }^{\circledR}\right)$, que foi aquecida até sua ebulição. Esta solução a $85^{\circ} \mathrm{C}$ foi adicionada à mistura em pó (polvilho doce/polvilho doce e polidextrose) para promover sua parcial gelatinização.

Após o resfriamento da massa escaldada à temperatura ambiente, foram adicionados os ovos, em seguida, acrescentou-se o queijo parmesão ralado (Piracanjuba ${ }^{\circledR}$ ) sendo incorporados por aproximadamente 10 minutos. As massas foram porcionadas em unidades de $15 \mathrm{~g}$ e moldadas manualmente até que os pães de queijo adquirissem um formato redondo.

Os pães de queijo foram dispostos em assadeiras de alumínio e em seguida foram congelados lentamente (cerca de $4 \mathrm{~h}$ ) em freezer à temperatura aproximada de $-18^{\circ} \mathrm{C}$. Após o congelamento, os pães de queijo foram acondicionados em sacos de plásticos e armazenados em freezer convencional até o momento do assamento e posteriores análises. $\mathrm{O}$ assamento dos pães de queijo congelados foi realizado utilizando forno elétrico preaquecido à temperatura de $200{ }^{\circ} \mathrm{C}$ por 20 minutos.

\subsection{Análises microbiológicas}

Foram realizadas análises microbiológicas nos pães de queijo após serem assados e resfriados a $25^{\circ} \mathrm{C}$ por 20 minutos. As análises microbiológicas foram: Staphylococcus coagulase positiva, Salmonella sp., Bacillus cereus e coliformes a $45^{\circ} \mathrm{C}$, todas em duplicata, nas formulações 1, 2, 3 e 4, seguindo as metodologias de Silva et al. (2010) descritas no "Manual de Métodos de Análise Microbiológica de Alimentos e Água". 


\subsection{Análise sensorial}

A análise sensorial foi realizada com acadêmicos da UFMS, que aceitaram participar da pesquisa mediante a assinatura do Termo de Consentimento Livre e Esclarecido (TCLE). Participaram 100 provadores não treinados como descrito por Macfie et al. (1989), adultos, de ambos os sexos, com idade superior a 18 anos. Como critérios de exclusão, foram considerados os seguintes fatores: não ser acadêmico da UFMS, menores de 18 anos, quilombolas, indígenas e indivíduos alérgicos à proteína do leite de vaca ou intolerantes à lactose.

Durante os julgamentos sensoriais dos produtos, os atributos avaliados foram: aceitação global, aparência, aroma, sabor e textura. Os provadores avaliaram a aceitação das amostras através da escala hedônica estruturada de 9 pontos ( 1 = desgostei muitíssimo, 9 = gostei muitíssimo), segundo Dutcosky (2013). Também foi aplicado o teste de intenção de compra analisado por meio de uma escala hedônica estruturada de 5 pontos ( 1 = certamente não compraria, 5 = certamente compraria), como sugerido por Minim (2013).

Cada julgador recebeu uma unidade de cada amostra assada com aproximadamente $15 \mathrm{~g}$, em copos plásticos brancos, codificados com números de três dígitos, de forma casualizada, acompanhados de um copo de água para o enxague da boca entre as análises. As formulações foram oferecidas aos julgadores de forma monádica sequencial, com o auxílio do pesquisador.

\subsection{Análises físico-químicas}

As análises físico-químicas foram realizadas em triplicata somente na amostra padrão e na amostra que apresentou maior aceitabilidade e maior teor de polidextrose, critério adotado baseado na literatura (Micheletti et al., 2018). A determinação da umidade foi realizada de acordo com Analysis of Association of Official Analytical Chemists, que consiste em secagem a $105^{\circ} \mathrm{C}$ até peso constante (Analysis of Association of Official Analytical Chemists, 2011). A determinação de cinzas foi realizada em mufla a $550{ }^{\circ} \mathrm{C}$, conforme metodologia de Analysis of Association of Official Analytical Chemists (1980). A determinação de proteína foi realizada através da avaliação do nitrogênio total da amostra, pelo método micro Kjeldahl com fator de conversão de nitrogênio para proteína de 6,25 (Analysis of Association of Official Analytical Chemists, 1980). A determinação de lipídeos foi realizada pelo método de Bligh \& Dyer (1959).

A determinação de carboidratos foi realizada por meio de cálculo teórico (por diferença) nos resultados, incluindo fibra, conforme a fórmula: $\%$ Carboidratos $=100-(\%$ umidade $+\%$ proteína $+\%$ lipídeos $+\%$ cinzas). O valor calórico (kcal) foi calculado em relação a $100 \mathrm{~g}$ da amostra, utilizando os valores Atwater (ou calor de combustão) para gordura $(9 \mathrm{kcal} / \mathrm{g})$, proteína $(4 \mathrm{kcal} / \mathrm{g})$ e carboidratos $(4 \mathrm{kcal} / \mathrm{g})$ (Atwater \& Woods, 1896).

A determinação do teor de fibra alimentar foi realizada através do cálculo teórico, levando-se em consideração a Tabela de Composição de Alimentos (Universidade Estadual de Campinas, 2011) e o laudo técnico da empresa que relatou que a polidextrose utilizada possui $90 \%$ de fibra alimentar total. Considerouse que os demais ingredientes não contribuíram para o teor de fibra alimentar.

\subsection{Determinação do Valor Diário de Referência (VD)}

O VD foi calculado em relação a $50 \mathrm{~g}$, com base nos valores médios preconizados para adultos de 18 a 70 anos (Dietary Reference Intakes, 2005), resultando em: 2021,73 kcal/dia, 260,11 g/dia de carboidratos, $72,57 \mathrm{~g} /$ dia de proteínas, 73,5 g/dia de lipídeos e 14,04 g/dia de fibra alimentar. 


\subsection{Análises físicas}

\subsubsection{Análise de textura instrumental e volume específico}

Os pães de queijo foram assados em forno preaquecido por 20 minutos à temperatura de $200{ }^{\circ} \mathrm{C}$. Em seguida, foram resfriados por 10 minutos em temperatura ambiente. A análise de textura (firmeza instrumental) foi realizada utilizando-se o texturômetro TA.XT2i (Stable Micro Systems, Haslemere, GBR) e probe SMS P/36R, segundo o método 74-09.01 da American Association of Cereal Chemists International (2010). Os parâmetros utilizados nos testes foram: velocidade pré-teste $=1,0 \mathrm{~mm} / \mathrm{s}$; velocidade de teste $=1,7 \mathrm{~mm} / \mathrm{s}$; velocidade pós teste $=10,0 \mathrm{~mm} / \mathrm{s}$; distância $40 \mathrm{~mm}$, com medida de força em compressão. Os resultados foram expressos em $\mathrm{fg}$ (força.grama) e representam a média de 3 determinações.

A propriedade de expansão foi avaliada através do volume específico $\left(\mathrm{cm}^{3} \mathrm{~g}^{-1}\right)$, obtido pela razão entre o volume aparente $\left(\mathrm{cm}^{3}\right)$, determinado pelo deslocamento das sementes de painço, e a massa (g), após o forneamento, aferido em proveta $50 \mathrm{~cm}^{3}$ (Pizzinatto et al., 1993).

\subsubsection{Análise de cor instrumental}

Para esta análise os pães de queijo foram assados e resfriados conforme descrito anteriormente na análise de textura instrumental. A análise de cor foi realizada da casca e do miolo utilizando-se colorímetro portátil modelo CR-410 (Konica Minolta, Japão), com o sistema CIE L*a*b*, onde L* indica a luminosidade e a* e $\mathrm{b}^{*}$ indicam as coordenadas de cromaticidade (Minolta, 2002). Os resultados representam a média de 3 determinações.

\subsection{Análise estatística}

Os dados da análise sensorial, textura e cor foram avaliados por meio da análise de variância (ANOVA), seguido do teste post-hoc de Tukey. Já o teste de $t$ de student foi aplicado para avaliação dos dados da composição físico-química dos produtos. Ambos os testes foram analisados com nível de 5\% de significância, com auxílio do software Statistical Package for the Social Sciences (SPSS), versão 22.0.

\section{Resultados e discussões}

\subsection{Análises microbiológicas}

Os resultados das análises microbiológicas estão descritos na Tabela 2.

Tabela 2. Resultados das análises microbiológicas das formulações de pão de queijo padrão e adicionadas de polidextrose.

\begin{tabular}{cccccc}
\hline Análises & $\begin{array}{c}\text { Limites } \\
\text { permitidos }\end{array}$ & F1 & F2 & F3 & F4 \\
\hline Staphylococcus & $5 \times 10^{2} \mathrm{UFC}^{* *} / \mathrm{g}$ & $<10^{1} \mathrm{UFC} / \mathrm{g}$ & $<10^{1} \mathrm{UFC} / \mathrm{g}$ & $<10^{1} \mathrm{UFC} / \mathrm{g}$ & $<10^{1} \mathrm{UFC} / \mathrm{g}$ \\
\hline Salmonella sp. & Ausência & Ausência & Ausência & Ausência & Ausência \\
\hline Bacillus cereus & $5 \times 10^{2} \mathrm{UFC} / \mathrm{g}$ & $3,0 \times 10^{1} \mathrm{UFC} / \mathrm{g}$ & $5,0 \times 10^{1} \mathrm{UFC} / \mathrm{g}$ & $2,0 \times 10^{1} \mathrm{UFC} / \mathrm{g}$ & $5,0 \times 10^{1} \mathrm{UFC} / \mathrm{g}$ \\
\hline Colif. a $45^{\circ} \mathrm{C}$ & $5 \times 10 \mathrm{UFC} / \mathrm{g}$ & $\mathrm{NMP} * * * \mathrm{~g}=<$ & $\mathrm{NMP} / \mathrm{g}=<3$ & $\mathrm{NMP} / \mathrm{g}=<3$ & $\mathrm{NMP} / \mathrm{g}=<3$ \\
\hline
\end{tabular}

*Resolução-RDC $\mathrm{N}^{\circ}$ 12, de 2 de janeiro de 2001 (Brasil, 2001). **UFC: Unidades Formadoras de Colônias por grama. ***NMP: Número Mais Provável por grama. F1: Padrão. F2: 2\% de polidextrose. F3: 4\% de polidextrose. F4: 6\% de polidextrose. 
Os resultados demonstraram que as formulações apresentaram qualidade microbiológica satisfatória, com contagens inferiores aos limites máximos estabelecidos pela RDC No 12 para todas as análises, indicando que não houve matérias-primas contaminadas e que não ocorreram falhas nas boas práticas de higiene e manipulação durante a elaboração e manipulação dos pães de queijo (Brasil, 2001).

\subsection{Análise sensorial}

Os resultados da análise sensorial das formulações de pão de queijo estão apresentados na Tabela 3.

Tabela 3. Médias dos testes sensoriais afetivos de aceitação e intenção de compra realizados para as formulações de pão de queijo padrão (F1) e adicionado de $2 \%$ (F2), $4 \%$ (F3) e $6 \%$ (F4) de polidextrose.

\begin{tabular}{|c|c|c|c|c|c|}
\hline \multirow{2}{*}{ Formulações/Atributos } & \multirow{2}{*}{$\frac{\text { F1 }}{\text { Média } \pm D P}$} & \multirow{2}{*}{$\frac{\text { F2 }}{\text { Média } \pm D P}$} & \multirow{2}{*}{$\frac{\text { F3 }}{\text { Média } \pm D P}$} & \multirow{2}{*}{$\frac{\text { F4 }}{\text { Média } \pm D P}$} & \multirow{2}{*}{$p$-valor } \\
\hline & & & & & \\
\hline Aparência ${ }^{\text {ns }}$ & $7,50 \pm 1,39$ & $7,67 \pm 1,30$ & $7,26 \pm 1,47$ & $7,28 \pm 1,58$ & 0,141 \\
\hline IA $(\%)$ & 83,3 & 85,2 & 80,6 & 80,8 & \\
\hline Aroma & $6,96 \pm 1,62^{b}$ & $7,73 \pm 1,06^{\mathrm{a}}$ & $7,33 \pm 1,44^{\mathrm{ab}}$ & $7,36 \pm 1,37^{\mathrm{ab}}$ & 0,002 \\
\hline IA $(\%)$ & 77,3 & 85,8 & 81,4 & 81,7 & \\
\hline Sabor & $6,45 \pm 1,88^{b}$ & $7,63 \pm 1,44^{\mathrm{a}}$ & $6,93 \pm 1,83^{\mathrm{bc}}$ & $6,80 \pm 1,65^{\mathrm{bc}}$ & $<0,0001$ \\
\hline IA $(\%)$ & 71,6 & 84,7 & 77,0 & 75,5 & \\
\hline Textura & $6,75 \pm 1,8^{\mathrm{b}}$ & $7,42 \pm 1,43^{\mathrm{a}}$ & $6,97 \pm 1,63^{\mathrm{ab}}$ & $6,69 \pm 1,70^{\mathrm{b}}$ & 0,008 \\
\hline IA $(\%)$ & 75,0 & 82,4 & 77,4 & 74,3 & \\
\hline Cor $^{\mathrm{ns}}$ & $7,50 \pm 1,43$ & $7,81 \pm 1,14$ & $7,40 \pm 1,34$ & $7,51 \pm 1,39$ & 0,154 \\
\hline IA (\%) & 83,3 & 86,7 & 82,2 & 83,4 & \\
\hline Aceitação Global & $6,70 \pm 1,68^{b}$ & $7,69 \pm 1,14^{\mathrm{a}}$ & $7,00 \pm 1,56^{\mathrm{b}}$ & $7,06 \pm 1,48^{\mathrm{b}}$ & $<0,0001$ \\
\hline IA ( $(\%)$ & 74,4 & 85,4 & 77,7 & 78,4 & \\
\hline Intenção de compra & $3,38 \pm 1,32^{\mathrm{b}}$ & $4,25 \pm 0,89^{\mathrm{a}}$ & $3,76 \pm 1,04^{\mathrm{b}}$ & $3,63 \pm 1,13^{\mathrm{b}}$ & $<0,0001$ \\
\hline IA (\%) & 67,6 & 85,0 & 75,2 & 72,6 & \\
\hline
\end{tabular}

${ }^{\text {ns }}$ nao significativo. Letras diferentes na linha indicam diferença significativa pelo teste post-hoc de Tukey $(p<0,05)$. DP: Desvio padrão. F1: Padrão. F2: 2\% de polidextrose. F3: 4\% de polidextrose. F4: $6 \%$ de polidextrose. IA: Índice de Aceitabilidade.

Não houve diferença significativa entre as formulações nos atributos aparência e cor $(p>0,05)$, demonstrando que as formulações não apresentaram características visuais para que os provadores pudessem identificar diferenças entre a formulação padrão e as formulações modificadas. Resultados similares foram observados por Montenegro et al. (2008), que constataram que visualmente a inclusão de polidextrose em biscoitos de polvilho azedo não modificou a cor do produto final.

Contudo, no atributo textura houve maior aceitação das formulações F2 e F3, demonstrando que a adição de níveis de até $4 \%$ de polidextrose obteve boa aceitação neste atributo. A redução da nota em F4 é aceitável, pois a adição de fibras pode aumentar a rigidez de produtos alimentícios e consequentemente alterar a textura (Izzo \& Niness, 2001).

Para um produto ser considerado aceito sensorialmente, é necessário que o percentual de aceitabilidade seja acima de 70\% (Teixeira et al., 1987; Dutcosky, 2013). Em todos os atributos avaliados as 4 formulações apresentaram índice de aceitabilidade superior a $70 \%$, exceto o atributo intenção de compra da formulação padrão. Sendo assim, esses resultados indicam que a expectativa de compra dos pães de queijo adicionados de polidextrose foi superior ao pão de queijo padrão. 
Notou-se que a formulação padrão apresentou índice de aceitabilidade inferior às formulações 2,3 e 4 nos atributos aroma, sabor, aceitação global e intenção de compra. Foram identificadas duas hipóteses para esses índices. A primeira hipótese é de que o estudo tenha desenvolvido um produto contendo fibras e com características mais agradáveis aos provadores comparativamente ao produto padrão sem adição de fibras.

Os bons índices de aceitabilidade podem ser atribuídos ao fato de que a adição de fibra pode contribuir para redução no percentual de gordura, sem causar grandes alterações no aroma ou sabor do produto final (Camargo et al., 2016; Cavalcante et al., 2016). Atualmente os consumidores aceitam a adição de ingredientes menos comuns a preparações tradicionais. Além disso, buscam opções mais saudáveis de alimentos amplamente consumidos, como é o caso do pão de queijo (Fell et al., 2017; Diório et al., 2018).

A segunda hipótese está relacionada à temperatura em que foram servidas as amostras aos provadores. $\mathrm{O}$ pão de queijo é um tipo de alimento cujo consumidor espera consumi-lo logo após sua retirada do forno, fato que não ocorreu neste estudo, para todas as amostras. Assim, embora não tenha sido mensurada a temperatura das amostras no momento da análise sensorial acredita-se que a F1 apresentou temperatura inferior às demais, o que poderia explicar índices de aceitabilidade menores ao esperado.

\subsection{Análises físico-químicas e Valor Diário de Referência (VD)}

As análises físico-químicas foram realizadas em triplicata, na formulação F1 (padrão) e F3 (4\% de polidextrose) devido aos resultados da aceitação sensorial semelhantes. Na Tabela 4, observa-se a composição físico-química e valores diários recomendados (VD) das formulações pão de queijo padrão e acrescidas de $4 \%$ de polidextrose, comparadas com um produto de referência.

Tabela 4. Composição físico-química e valores diários recomendados - VD* (porção média de 50 gramas) das formulações F1 (padrão) e F3 (4\% de polidextrose), comparados com um produto referência.

\begin{tabular}{|c|c|c|c|c|c|}
\hline \multirow{2}{*}{ Avaliação } & \multicolumn{2}{|l|}{ F1 } & \multicolumn{2}{|c|}{ F3 } & \multirow{2}{*}{ Referência** } \\
\hline & Média \pm DP & VD (\%)* & Média $\pm D P$ & VD (\%)* & \\
\hline Umidade (\%) ${ }^{\text {ns }}$ & $20,03 \pm 0,31$ & ND & $19,60 \pm 0,40$ & ND & 33,70 \\
\hline Cinzas $(\mathrm{RMF})\left({\left.\mathrm{g} 100 \mathrm{~g}^{-1}\right) * * *}^{*}\right.$ & $2,86 \pm 0,05^{b}$ & ND & $4,03 \pm 0,04^{\mathrm{a}}$ & ND & 2,30 \\
\hline Proteínas $\left({\left.\mathrm{g} 100 \mathrm{~g}^{-1}\right) * * *}^{* *}\right.$ & $9,44 \pm 0,51^{b}$ & 6,5 & $11,14 \pm 0,42^{\mathrm{a}}$ & 7,37 & 5,10 \\
\hline Lipídeos $\left(\mathrm{g} 100 \mathrm{~g}^{-1}\right)^{* * *}$ & $15,06 \pm 0,46^{\mathrm{a}}$ & 10,25 & $12,32 \pm 0,38^{\mathrm{b}}$ & 8,38 & 24,60 \\
\hline 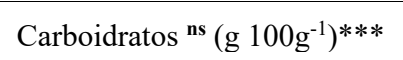 & $52,34 \pm 0,14$ & 10,06 & $52,79 \pm 1,34$ & 10,15 & 34,20 \\
\hline Calorias $\left(\mathrm{kcal}^{\left.100 \mathrm{~g}^{-1}\right) * * *}\right.$ & $383,35 \pm 3,17^{\mathrm{a}}$ & 9,48 & $366,95 \pm 0,32^{b}$ & 9,08 & 363,00 \\
\hline Fibra alimentar $\left({\left.\mathrm{g} 100 \mathrm{~g}^{-1}\right) * * * *}^{*}\right.$ & 0,00 & 0,00 & 3,6 & 12,82 & 0,60 \\
\hline
\end{tabular}

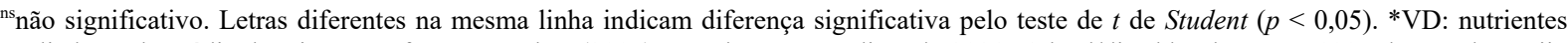
avaliados pela média da Dietary Reference Intakes (2005), com base numa dieta de 2021,73 kcal/dia. **Valores em 100g de pão de queijo assado (Universidade Estadual de Campinas, 2011). ***Valores calculados em base úmida. ****Cálculo teórico. DP: desvio padrão da média. RMF: resíduo mineral fixo. ND: não disponível.

Os resultados demostraram que não houve diferença $(p>0,05)$ nos teores de umidade entre a F1 e a F3. Isso pode ser explicado em virtude da polidextrose ser uma fibra solúvel que possui a capacidade de incorporação de água, proporcionando consistência à massa, evitando a perda de umidade (Achour et al., 1994; Pinheiro \& Penna, 2004). Porém, as amostras apresentaram umidade acima do preconizado pela RDC $\mathrm{n}^{\circ} 263$, que define um máximo de $18 \%$ de umidade em produtos de panificação a base de amido ou fécula de mandioca (Brasil, 2005).

Também não houve diferença $(p>0,05)$ no teor de carboidratos, o que era esperado, em virtude de as formulações terem sido elaboradas com as mesmas quantidades de polvilho e de leite, sendo os ingredientes que mais contribuíram como fonte de carboidratos. Em relação aos teores de resíduo mineral fixo (cinzas) e de proteínas, acredita-se que o 
preparo manual das amostras possa ter comprometido a homogeneização dos ingredientes, especialmente do queijo ralado, o que poderia explicar teores de cinzas e proteínas superiores na F3 $(p<0,05)$.

Segundo a $\mathrm{RDC} \mathrm{n}^{\circ} 54$, para que um alimento seja considerado com teor reduzido ou light em valor energético ou em algum nutriente é necessária uma redução de, no mínimo, 25\% em relação ao alimento de referência ou convencional (Brasil, 2012). Embora o objetivo do estudo não fosse desenvolver uma formulação padrão com teor reduzido de lipídeos, observou-se que tanto a formulação padrão F1 quanto a formulação $\mathrm{F} 3$ foram consideradas como produtos light, pois obtiveram redução de 38,78 e 49,92\% de lipídeos, respectivamente, quando comparadas ao pão de queijo de referência (Universidade Estadual de Campinas, 2011).

Vale a pena ressaltar que a redução nos teores de lipídeos da F3 mostrou estatisticamente $(p<0,05)$ uma redução de 4,28\% do seu valor calórico quando comparada à formulação padrão. Sabe-se que é um desafio tecnológico o desenvolvimento de produtos de panificação com redução do valor calórico, ao passo que ingredientes como a gordura lhes conferem importantes características de textura, maciez, sabor e conservação (Benassi et al., 2001).

Segundo a legislação brasileira, o termo "Fonte de Fibras" só pode ser utilizado nos rótulos nutricionais quando o alimento pronto (sólido) para o consumo apresentar no mínimo $3 \mathrm{~g}$ de fibras/100 g (Brasil, 2012). Embora a pesquisa realizada não pôde comprovar tais valores, segundo os cálculos aplicados, acredita-se que a formulação F3 apresentou teor de 3,6 g/ $100 \mathrm{~g}$ de fibras em base úmida, representando um aumento significativo em relação à formulação padrão, tornando-a como possível "Fonte de Fibras", devido principalmente, ao alto teor de fibras presente na polidextrose $(90 \mathrm{~g} / 100 \mathrm{~g})$.

Em uma população adulta e saudável na faixa de 18 a 70 anos, o consumo de uma porção de $50 \mathrm{~g}$ do pão de queijo contendo 3,6 $\mathrm{g}$ de fibras atingiria 12,82\% de suas necessidades diárias de fibras (Dietary Reference Intakes, 2005). As fibras solúveis, por não serem digeridas pelas enzimas do trato digestivo humano, estimulam o crescimento e a atividade de bactérias intestinais promotoras de saúde, especialmente as bifidobactérias (Gibson \& Roberfroid, 1995). Alguns estudos já demonstraram que o consumo adequado de fibras diariamente, pode contribuir com benefícios à saúde (Bernaud \& Rodrigues, 2013; Ibarra et al., 2015; Camargo et al., 2016).

As fibras possuem baixo valor calórico e a polidextrose, como já demonstrado em estudos, pode influenciar positivamente na absorção dos minerais cálcio, ferro, magnésio e fósforo, além de ajudar no controle do apetite, promovendo saciedade, o que a longo prazo poderia trazer benefícios à saúde, com o controle e a prevenção de doenças como a obesidade, diabetes mellitus tipo 2, hipertensão arterial e dislipidemias (Gibson et al., 2004; Santos et al., 2011; Tiihonen et al., 2011; Konings et al., 2014; Carmo et al., 2016).

\subsection{Análises físicas}

\subsubsection{Análise de textura instrumental e volume específico}

Os resultados de textura e volume específico dos pães de queijo estão apresentados na Tabela 5.

Tabela 5. Resultados de textura (firmeza) instrumental e volume específico dos pães de queijo.

\begin{tabular}{|c|c|c|c|c|c|}
\hline & Média \pm DP & Média $\pm D P$ & Média \pm DP & Média \pm DP & \\
\hline & F1 & F2 & F3 & F4 & \\
\hline Textura (f g) & $4333,11 \pm 509,09^{b}$ & $4888,54 \pm 1031,46^{\mathrm{b}}$ & $5478,49 \pm 961,48^{b}$ & $10063,44 \pm 1252,50^{\mathrm{a}}$ & $<0,0001$ \\
\hline $\begin{array}{l}\text { Volume Específico } \\
\qquad\left(\mathrm{cm}^{3} \mathrm{~g}^{-1}\right)\end{array}$ & $1,45 \pm 0,0^{\mathrm{a}}$ & $1,37 \pm 0,02^{\mathrm{b}}$ & $1,32 \pm 0,00^{\mathrm{c}}$ & $1,20 \pm 0,01^{\mathrm{d}}$ & $<0,001$ \\
\hline
\end{tabular}

Letras diferentes na linha indicam diferença significativa pelo teste post-hoc de Tukey $(p<0,05)$. DP: Desvio padrão. F1: Padrão. F2: $2 \%$ de polidextrose. F3: 4\% de polidextrose. F4: 6\% de polidextrose. 
O pão de queijo possui estrutura alveolar, formada pela expansão e evaporação dos líquidos presentes na massa, apresenta firmeza intermediária e massa menos elástica característica de gel formado pela gelatinização do amido (polvilho) e interação com proteínas (queijo e ovos) (Esteller \& Lannes, 2005). Os resultados demonstraram que não houve diferença significativa entre F1, F2 e F3 em relação à firmeza, apenas a F4 apresentou diferença estatisticamente significativa $(p<0,05)$ na textura em relação às demais (Tabela 5).

Segundo Anjos et al. (2014), a força máxima avaliada para produtos panificados é dependente da formulação, umidade da massa e conservação, e quanto menor o valor de firmeza, maior a maciez do produto, ou seja, a adição de polidextrose não modificou $(p>0,05)$ a maciez dos pães de queijo nas formulações F2 e $\mathrm{F} 3$, comparadas à padrão (F1), corroborando os relatos dos provadores na análise sensorial (Tabela 3 ).

Sabe-se que a gordura afeta a textura do pão de queijo por atuar como emulsificante, contribuindo para maior retenção de água, elasticidade e melhor textura (Maistro, 1999; Minim et al., 2000). Pode-se notar que a adição de $4 \%$ de polidextrose não alterou a textura dos pães de queijo, demonstrando que até neste percentual de adição, a fibra desempenhou papel semelhante a gordura, sendo justificado pelo fato de que as fibras solúveis tendem a formar géis em contato com a água auxiliando no aumento de retenção de umidade, promovendo a maciez em produtos de panificação (Maier, 1993).

O aumento nos níveis de polidextrose diminuiu o volume específico dos pães (Tabela 5), mostrando diferenças estatísticas $(p<0,05)$. O volume específico é importante na determinação da qualidade, porque é influenciado pelos ingredientes usados na formulação, sendo expresso pela relação entre o volume e a massa do produto após o forneamento. Pães de queijo com volume específico baixo podem apresentar um aspecto desagradável ao consumidor (Zavareze et al., 2009; Lemos et al., 2012).

A adição de polidextrose e a redução do óleo de soja interferiram no volume específico dos pães de queijo, devido ao fato de que a gordura também é importante para a propriedade de expansão, atua como um lubrificante molecular ajudando a massa a ter maior extensibilidade, contribuindo para maior elasticidade e melhor textura do miolo (Pereira et al., 2004).

\subsubsection{Análise de cor}

Os resultados de cor dos pães de queijo estão apresentados na Tabela 6.

Tabela 6. Resultado de cor da casca e do miolo dos pães de queijo.

\begin{tabular}{|c|c|c|c|c|c|}
\hline & Média $\pm D P$ & Média $\pm D P$ & Média $\pm D P$ & Média $\pm D P$ & \\
\hline & F1 & F2 & F3 & F4 & \\
\hline \multicolumn{6}{|l|}{ Casca } \\
\hline $\mathbf{L}^{* \mathrm{~ns}}$ & $34,34 \pm 0,46$ & $34,71 \pm 0,67$ & $35,18 \pm 0,54$ & $35,41 \pm 0,43$ & 0,091 \\
\hline$a^{*}$ & $2,13 \pm 0,41^{\mathrm{b}}$ & $2,24 \pm 0,71^{\mathrm{bc}}$ & $3,09 \pm 0,60^{\mathrm{bc}}$ & $3,51 \pm 050^{\mathrm{ac}}$ & 0,034 \\
\hline $\mathbf{b}^{*}$ & $9,60 \pm 0,23^{b}$ & $9,81 \pm 0,87^{\mathrm{bc}}$ & $10,09 \pm 1,17^{\mathrm{bc}}$ & $11,16 \pm 0,54^{\mathrm{ac}}$ & 0,024 \\
\hline \multicolumn{6}{|l|}{ Miolo } \\
\hline$L^{* n s}$ & $30,40 \pm 0,24$ & $30,60 \pm 0,26$ & $30,52 \pm 0,43$ & $30,53 \pm 0,47$ & 0,904 \\
\hline$a^{* \text { ns }}$ & $0,67 \pm 0,24$ & $0,59 \pm 0,13$ & $0,53 \pm 0,07$ & $0,44 \pm 0,17$ & 0,292 \\
\hline $\mathbf{b}^{* \mathrm{~ns}}$ & $7,11 \pm 0,48$ & $7,48 \pm 0,23$ & $7,79 \pm 0,64$ & $7,92 \pm 0,34$ & 0,106 \\
\hline
\end{tabular}

${ }^{\mathrm{n} s}$ não significativo. Letras diferentes na linha indicam diferença significativa pelo teste post-hoc de Tukey $(p<0,05)$. DP: Desvio padrão. F1: Padrão. F2: 2\% de polidextrose. F3: 4\% de polidextrose. F4: 6\% de polidextrose.

A luminosidade $\left(\mathrm{L}^{*}\right)$ pode ser definida como a capacidade de um objeto refletir ou transmitir luz resultando em uma cor luminosa ( 100 - branco) ou escura $(0$ - preto) e a cromaticidade é composta pelas coordenadas 
$a^{*}$ (vermelho-verde: valor positivo representa vermelho e negativo, verde) e b* (amarelo-azul: valor positivo representa amarelo e negativo, azul) (Arias et al., 2000; Minolta, 2002).

Os resultados das médias dos valores de $L^{*}$ da casca e do miolo não obtiveram diferença significativa ( $p$ $>0,05)$ demonstrando que as formulações com incorporação de polidextrose mantiveram capacidade semelhante a padrão em transmitir luz. Em relação aos valores médios de $a^{*}$ e b* da casca, foi possível observar que as amostras apresentaram cromaticidades diferentes $(p<0,05)$, com tons de cores que variaram de vermelho até amarelo (valores positivos).

Durante a elaboração dos pães de queijo (Figura 1) e na análise sensorial (Tabela 3) não foram observadas modificações na cor. Entretanto, segundo Peressini \& Sensidoni (2009), a utilização de porcentagens elevadas de fibra pode desencadear a reação de Maillard, ocasionando o escurecimento não enzimático do alimento, o que justificaria a diferença $(p<0,05)$ de cromaticidade na casca entre as amostras.

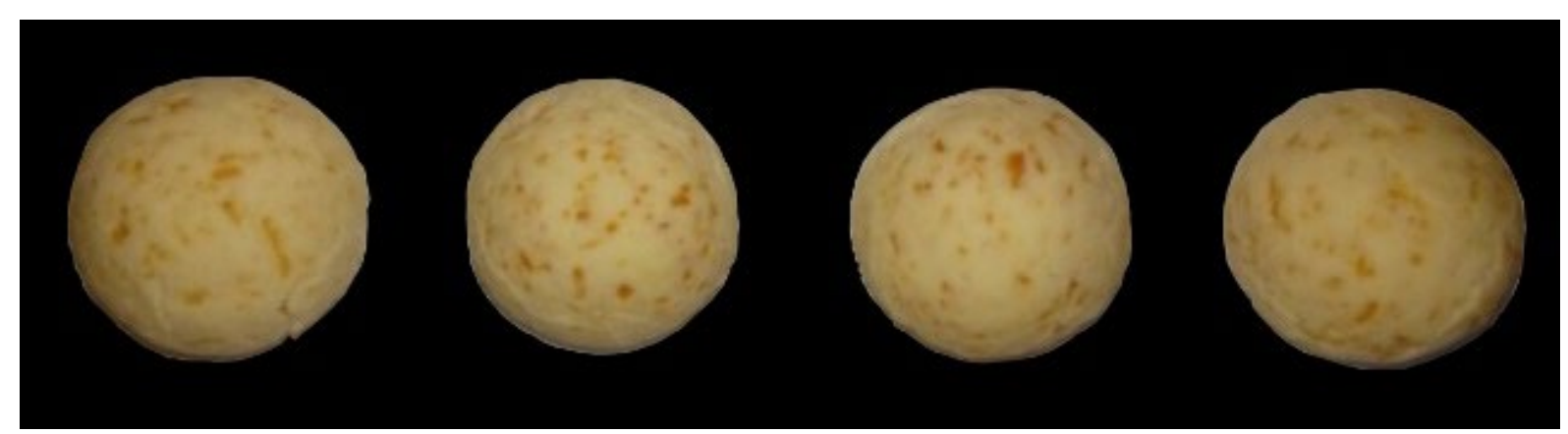

Figura 1. Pães de queijo formulados com diferentes concentrações de polidextrose. Figura da esquerda para a direita tem-se: F1 (Padrão): 0\% de polidextrose. F2: 2\% de polidextrose. F3: 4\% de polidextrose. F4: 6\% de polidextrose. Fonte: Os autores.

Já no miolo não foi observada diferença significativa $(p>0,05)$ entre as amostras nas coordenadas a* $\mathrm{e}$ $\mathrm{b}^{*}$, evidenciando que a polidextrose não alterou a cor das amostras quando comparadas à padrão. Além disso, a temperatura e a umidade presentes no miolo de produtos panificados podem impedir alteração significativa de cor. Sabe-se que valores mais altos de $\mathrm{b}^{*}$ em relação ao $\mathrm{a}^{*}$ são traduzidos para amostras com forte coloração amarelada ou dourada, que embora sejam menos aparentes quando comparadas à coloração castanha característica de produtos forneados, conseguem ser identificadas pelo colorímetro e aparecem em produtos como pão de queijo (Esteller \& Lannes, 2005).

\section{Conclusões}

A realização deste estudo com adição de polidextrose em pães de queijo permitiu a obtenção de formulações com menores teores de lipídeos e calorias, fonte de fibras e ótimas características físicas que indicam seu potencial de comercialização. A elaboração dos produtos permitiu comprovar que um nível de adição de até $4 \%$ de polidextrose em pães de queijo em substituição à gordura obteve aceitação sensorial e propriedades tecnológicas semelhantes ao produto padrão. Os resultados sugerem a possibilidade de novos estudos, visando o desenvolvimento, aprimoramento e a produção de alimentos funcionais saborosos, que poderão ser incluídos na alimentação de consumidores preocupados com a saúde.

\section{Referências}

Achour, L., Flourié, B., Briet, F., Pellier, P., Marteau, P., \& Rambaud, J. C. (1994). Gastrointestinal effects and energy value of polydextrose in healthy nonobese men. The American Journal of Clinical Nutrition, 59(6), 1362-1368. PMid:8198061. http://dx.doi.org/10.1093/ajcn/59.6.1362

American Association of Cereal Chemists International - AACC. (2010). Approved methods (11th ed.). St. Paul: AACC. 
Analysis of Association of Official Analytical Chemists - AOAC. (1980). Official methods of analysis of Association of Official Analytical Chemists (13th ed.). Washington: AOAC.

Analysis of Association of Official Analytical Chemists - AOAC. (2011). Official methods of analysis of Association of Official Analytical Chemists (18th ed.). Gaithersburg: AOAC

Anjos, L. D., Pereira, J., Couto, E. M., \& Cirillo, M. A. (2014). Modified starches or stabilizers in preparation of cheese bread. Ciência Rural, 44(9), 1686-1691. http://dx.doi.org/10.1590/0103-8478cr20131133

Arias, R., Lee, T. C., Logendra, L., \& Janes, H. (2000). Correlation of lycopene measured by HPLC with the $L^{*}, a^{*}, b^{*}$ color readings of a hydroponic tomato and the relationship of maturity with color and lycopene content. Journal of Agricultural and Food Chemistry, 48(5), 1697-1702. PMid:10820081. http://dx.doi.org/10.1021/jf990974e

Atwater, W. O., \& Woods, C. D. (1896). The chemical composition of American food materials (Experiment Station Bulletin, Vol. 1, No. 28, 51 p.). Washington: US Official Experiment Stations.

Benassi, V. T., Watanabe, E., \& Lobo, A. R. (2001). Produtos de panificação com conteúdo calórico reduzido. Boletim do Centro de Pesquisa e Processamento de Alimentos, 19(2), 225-242. http://dx.doi.org/10.5380/cep.v19i2.1235

Bernaud, F. S. R., \& Rodrigues, T. C. (2013). Fibra alimentar: Ingestão adequada e efeitos sobre a saúde do metabolismo. Arquivos Brasileiros de Endocrinologia \& Metabologia, 57(6), 397-405. PMid:24030179. http://dx.doi.org/10.1590/S000427302013000600001

Bligh, E. C., \& Dyer, W. J. (1959). A rapid method of total lipid extraction and purification. Canadian Journal of Biochemistry and Physiology, 37(8), 911-917. PMid:13671378. http://dx.doi.org/10.1139/059-099

Brasil. Ministério da Saúde. Agência Nacional de Vigilância Sanitária - ANVISA. (2001, janeiro 2). Aprova o Regulamento Técnico sobre padrões microbiológicos para alimentos (Resolução da Diretoria Colegiada $n^{\circ} 12$, de 2 de janeiro de 2001). Diário Oficial [da] República Federativa do Brasil, Brasília. Recuperado em 20 de abril de 2019, de http://bvsms.saude.gov.br/bvs/saudelegis/anvisa/2001/res0012_02_01_2001.html

Brasil. Ministério da Saúde. Agência Nacional de Vigilância Sanitária - ANVISA. (2005, setembro 22). Aprova o Regulamento técnico para produtos de cereais, amidos, farinhas e farelos (Resolução da Diretoria Colegiada n ${ }^{\circ} 263$, de 22 de setembro de 2005). Diário Oficial [da] República Federativa do Brasil, Brasília. Recuperado em 20 de abril de 2019, de http://bvsms.saude.gov.br/bvs/saudelegis/anvisa/2005/rdc0263_22_09_2005.html

Brasil. Ministério da Saúde. Agência Nacional de Vigilância Sanitária - ANVISA. (2012, novembro 12). Aprova o Regulamento Técnico sobre Informação Nutricional Complementar (Resolução da Diretoria Colegiada n 54, de 12 de novembro de 2012). Diário Oficial [da] República Federativa do Brasil, Brasília. Recuperado em 20 de abril de 2019, de

http://bvsms.saude.gov.br/bvs/saudelegis/anvisa/2012/rdc0054_12_11_2012.html

Camargo, A., Mendes, A. C. S., Mendes, L. A., \& Silva, F. C. (2016). Desenvolvimento de pão de queijo com baixo teor de sódio e enriquecido com fibras. Revista Intellectus, 3(35), 51-73.

Carmo, M. M. R., Walker, J. C. L., Novello, D., Caselato, V. M., Sgarbieri, V. C., Ouwehand, A. C., Andreollo, N. A., Hiane, P. A., \& Santos, E. F. (2016). Polydextrose: Physiological function, and effects on health. Nutrients, 8(9), 541-553. PMid:27618093. http://dx.doi.org/10.3390/nu8090553

Cavalcante, R. B. M., Morgano, M. A., Silva, K. J. D., Rocha, M. M., Araújo, M. A. M., \& Moreira-Araújo, R. S. R. (2016). Cheese bread enriched with biofortified cowpea flour. Ciência e Agrotecnologia, 40(1), 97-103. http://dx.doi.org/10.1590/S141370542016000100009

Dietary Reference Intakes - DRI. (2005). Dietary Reference Intakes for energy, carbohydrate, fiber, fat, fatty acids, cholesterol, protein and amino acids. Washington: The National Academies Press.

Diório, M. S., Silva, J. G., \& Teixeira, N. C. (2018). Pão de queijo saudável: Tradição e saúde caminhando juntos. Revista Pensar Gastronomia, 4(1), 1-25. Recuperado em 20 de abril de 2019, de http://revistapensar.com.br/gastronomia/pasta_upload/artigos/a80.pdf Dutcosky, S. D. (2013). Análise sensorial de alimentos (4. ed., 531 p.). Curitiba: Champagnat.

Esteller, M. S., \& Lannes, S. C. S. (2005). Parâmetros complementares para fixação de identidade e qualidade de produtos panificados. Food Science and Technology, 25(4), 802-806. http://dx.doi.org/10.1590/S0101-20612005000400028

Fell, J. T., Seidel, L. E., Pacheco, H. C., Kemerich, G. T., \& Oliveira, E. C. (2017). Elaboração e avaliação das características físicoquímicas de pães de queijo com diferentes bases. Revista Destaques Acadêmicos, 9(4), 151-160. http://dx.doi.org/10.22410/issn.2176-3070.v9i4a2017.1663

Fernandes, D. S., Del Bem, M. S., Sorroche, C., Leonel, M., \& Leonel, S. (2015). Elaboração de pão de queijo adicionado com farinha de banana verde: Características físicas e sensoriais. Revista Raízes e Amidos Tropicais, 11(1), 56-65. Recuperado em 20 de abril de 2019, de http://energia.fca.unesp.br/index.php/rat/article/view/2128

Gibson, G. R., \& Roberfroid, M. B. (1995). Dietary modulation of the human colonic microbiota: Introducing the concept of prebiotics. The Journal of Nutrition, 125(6), 1401-1412. PMid:7782892. http://dx.doi.org/10.1093/jn/125.6.1401

Gibson, G. R., Probert, H. M., Loo, J. V., Rastall, R. A., \& Roberfroid, M. B. (2004). Dietary modulation of the human colonic microbiota: Updating the concept of prebiotics. Nutrition Research Reviews, 17(2), 259-275. PMid:19079930. http://dx.doi.org/10.1079/NRR200479

Ibarra, A., Astbury, N. M., Olli, K., Alhoniemi, E., \& Tiihonen, K. (2015). Effects of polydextrose on different levels of energy intake: A systematic review and meta-analysis. Appetite, 87(1), 30-37. PMid:25510531. http://dx.doi.org/10.1016/j.appet.2014.12.099

Izzo, M., \& Niness, K. (2001). Formulating nutrition bars with inulin and oligofructose. Cereal Foods World, 46(3), 102-105.

Konings, E., Schoffelen, P. F., Stegen, J., \& Blaak, E. E. (2014). Effect of polydextrose and soluble maize fibre on energy metabolism, metabolic profile and appetite control in overweight men and women. British Journal of Nutrition, 111(1), 111-121. PMid:23880340. http://dx.doi.org/10.1017/S0007114513002183 
Lemos, A. R., Capriles, V. D., Pinto e Silva, M. E. M., \& Arêas, J. A. G. (2012). Effect of incorporation of amaranth on the physical properties and nutritional value of cheese bread. Food Science and Technology, 32(3), 427-431. http://dx.doi.org/10.1590/S010120612012005000079

Macfie, H. J., Bratchell, N., Greenhoff, K., \& Vallis, L. V. (1989). Designs to balance the effect of order of presentation and first-order carry-over effects in hall tests. Journal of Sensory Studies, 4(2), 129-148. http://dx.doi.org/10.1111/j.1745-459X.1989.tb00463.x

Machado, A. V., \& Pereira, J. (2010). Elaboração de pão de queijo enriquecido com fibras utilizando, farinha de linhaça dourada (linun isutatissimum I.) e farinha de berinjela (solanum melongena, I.). Ciência e Agrotecnologia, 34(2), 421-427.

Maier, H. (1993). Guar, locust bean, tara and fenugreek gums. In R. L. Whistler \& J. N. Bemiller (Eds.), Industrial gums: Polysaccharides and their derivatives (3. ed., Cap. 8, pp. 181-226). New York: Academic Press. http://dx.doi.org/10.1016/B978-008-092654-4.50012-7.

Maistro, L. C. (1999). Aplicação de concentrados proteicos de soro de leite bovino em iogurtes (Tese de doutorado). Faculdade de Engenharia de Alimentos, Universidade Estadual de Campinas, Campinas. Recuperado em 20 de abril de 2019, de http://repositorio.unicamp.br/jspui/handle/REPOSIP/254300

Micheletti, J., Soares, J. M., Franco, B. C., Carvalho, I. R. A., Candido, C. J., Santos, E. F., \& Novello, D. (2018). The addition of jaboticaba skin flour to muffins alters the physicochemical composition and their sensory acceptability by children. Brazilian Journal of Food Technology, 21(1), 1-8. http://dx.doi.org/10.1590/1981-6723.08917

Minim, V. P. R. (2013). Análise sensorial: Estudo com consumidores (3. ed). Viçosa: Universidade Federal de Viçosa.

Minim, V. P. R., Machado, P. T., Canavesi, E., \& Pirozi, M. R. (2000). Perfil sensorial e aceitabilidade de diferentes formulações de pão de queijo. Food Science and Technology, 20(2), 154-159. http://dx.doi.org/10.1590/S0101-20612000000200005

Minolta. (2002). User manual: Chroma Meter Modelo CR 410. New Jersey: Konica Minolta.

Montenegro, F. M., Gomes-Ruffi, C. R., Vicente, C. A., Collares-Queiroz, F. P., \& Steel, C. J. (2008). Biscoitos de polvilho azedo enriquecidos com fibras solúveis e insolúveis. Food Science and Technology, 28(1), 184-191. http://dx.doi.org/10.1590/S010120612008000500029

Pereira, J., Ciacco, C. F., Vilela, E. R., \& Pereira, R. G. F. A. (2004). Função dos ingredientes na consistência da massa e nas características do pão de queijo. Food Science and Technology, 24(4), 494-500. http://dx.doi.org/10.1590/S010120612004000400003

Peressini, D., \& Sensidoni, A. (2009). Effect of soluble dietary fibre addition on rheological and bread making properties of wheat doughs. Journal of Cereal Science, 49(2), 190-201. http://dx.doi.org/10.1016/j.jcs.2008.09.007

Pinheiro, M. V. S., \& Penna, A. L. B. (2004). Substitutos de gordura: Tipos e aplicações em produtos lácteos. Alimentos e Nutrição, 15(2), 175-186. Recuperado em 20 de abril de 2019, de http://servbib.fcfar.unesp.br/seer/index.php/alimentos/article/viewFile/72/87

Pinto, A. L. D., \& Paiva, C. L. (2010). Desenvolvimento de uma massa funcional pronta para tortas utilizando o método de Desdobramento da Função Qualidade (QFD). Food Science and Technology, 30(1), 36-43. http://dx.doi.org/10.1590/S010120612010000500007

Pizzinatto, A., Magno, C. P. R., \& Campagnolli, D. M. F. (1993). Avaliação tecnológica de produtos derivados da farinha de trigo (pão, macarrão, biscoitos) (54 p.). Campinas: Centro de Tecnologia de Farinhas e Panificação, Instituto de Tecnologia de Alimentos.

Santos, E. F., Tsuboi, K. H., Araújo, M. R., Falconi, M. A., Ouwehand, A. C., Andreollo, N. A., \& Miyasaka, C. K. (2010). Ingestion of polydextrose increase the iron absorption in rats submitted to partial gastrectomy. Arquivos Brasileiros de Cirurgia Digestiva, 25(1), 518-524. PMid:21120284.

Santos, E. F., Tsuboi, K. H., Araújo, M. R., Falconi, M. A., Ouwehand, A. C., Andreollo, N. A., \& Miyasaka, C. K. (2011). A ingestão de prebióticos previne a mal absorção de ferro e anemia induzidas pela gastrectomia? Estudo experimental em ratos. Arquivos Brasileiros de Cirurgia Digestiva, 24(1), 9-14. http://dx.doi.org/10.1590/S0102-67202011000100003

Silva, N., Junqueira, V. C. A., Silveira, N. F. A., Taniwaki, M. H., Santos, R. F. S., \& Gomes, R. A. R. (2010). Manual de métodos de análise microbiológica de alimentos e água (4. ed.). São Paulo: Ed. Varela. 624 p.

Teixeira, E., Meinert, E., \& Barbetta, P. A. (1987). Análise sensorial dos alimentos (180 p.). Florianópolis: Universidade Federal de Santa Catarina.

Tiihonen, K. K., Röytiö, H., Putaala, H., \& Ouwehand, A. C. (2011). Polydextrose functional fibre improving digestive health, satiety and beyond. Nutrafoods, 10(1), 23-28. http://dx.doi.org/10.1007/BF03223385

Universidade Estadual de Campinas - UNICAMP. (2011). Tabela Brasileira de Composição de Alimentos - TACO (4. ed. rev. ampl.,161 p.). Campinas: UNICAMP/NEPA. Recuperado em 20 de abril de 2019, de http://www.nepa.unicamp.br/taco/tabela.php?ativo=tabela

Zavareze, E. R., Storck, C. R., Pereira, J. M., Gularte, M. A., \& Dias, A. R. G. (2009). Elaboration of cheese bread with substitution of the cassava starch by sweet potato (Ipomoea batatas) starch and submitted to different drying processes. Brazilian Journal of Food Technology, 12(1), 68-76. http://dx.doi.org/10.4260/BJFT2009490800009 